\title{
Bon temps mauvais temps : Prévision automatisée de la cryptosporidiose saisonnière en Ontario à base d'apprentissage machine
}

\author{
Olaf Berke ${ }^{1 *}$, Lise Trotz-Williams ${ }^{1,2}$, Simon de Montigny $y^{3,4}$
}

\section{Résumé}

Contexte : L'augmentation de l'utilisation des mégadonnées et de la modélisation prédictive connexe fondée sur des algorithmes d'apprentissage automatique au cours des deux dernières décennies a fourni de nouvelles possibilités de surveillance des maladies et de préparation de la santé publique. Les mégadonnées s'accompagnent de la promesse d'une production et d'un accès plus rapide à des renseignements précis, ce qui pourrait faciliter la précision prédictive en santé publique (" santé publique de précision »). À titre d'exemple, nous avons envisagé de prévoir l'évolution future de l'incidence mensuelle de la cryptosporidiose en Ontario.

Méthodes : L'approche statistique traditionnelle en matière de prévision est le modèle de moyenne mobile intégrée saisonnière autorégressive (SARIMA). Nous avons appliqué le modèle SARIMA et une approche de réseau de neurones artificiels (RNA), plus précisément un réseau de neurones à propagation avant, pour prévoir l'incidence mensuelle de la cryptosporidiose en Ontario en 2017 en utilisant les données de 2005 à 2016 comme ensemble d'apprentissage initial. Les deux approches de prévision sont automatisées pour les rendre pertinentes au contexte de la surveillance des maladies. Nous avons comparé les prévisions résultantes en utilisant la racine de l'erreur quadratique moyenne (REQM) et l'erreur absolue moyenne (EAM) comme mesures de la précision des prévisions.

Résultats : La cryptosporidiose est une maladie saisonnière qui atteint son apogée en Ontario à la fin de l'été. Dans cette étude, le modèle SARIMA et les approches de prévision du RNA ont saisi le schéma saisonnier de la cryptosporidiose. Contrairement à des études semblables publiées dans la documentation scientifique, les prévisions du RNA sur la cryptosporidiose étaient légèrement moins précises que les prévisions du modèle SARIMA.

Conclusion : Les approches du RNA et du SARIMA conviennent à la prévision automatisée des données des séries chronologiques en santé publique provenant des systèmes de surveillance. Les études futures devraient utiliser des algorithmes supplémentaires (e.g. forêts d'arbres décisionnels) et évaluer l'exactitude en utilisant d'autres maladies comme études de cas et en réalisant des études de simulation rigoureuses. La différence entre les prévisions de l'algorithme d'apprentissage automatique, c.-à-d. le RNA, et le modèle d'apprentissage statistique, c.-à-d. le SARIMA, doit être prise en compte en ce qui concerne les différences philosophiques entre les deux approches.
Cette oeuvre est mise à la disposition selon les termes de la licence internationale Creative Commons Attribution 4.0

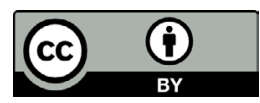

Affiliations

1 Department of Population Medicine, Collège de médecine vétérinaire de l'Ontario, Université de Guelph, Guelph, ON

2 Wellington-Dufferin Guelph Public Health, Guelph, ON

${ }^{3}$ École de santé publique Département de médecine sociale et préventive, Université de Montréal, Montréal, QC

${ }^{4}$ Centre de recherche du $\mathrm{CHU}$ Sainte-Justine, Montréal, QC

\section{${ }^{\star}$ Correspondance :} oberke@uoguelph.ca
Citation proposée : Berke O, Trotz-Williams L, de Montigny S. Bon temps mauvais temps : Prévision automatisée de la cryptosporidiose saisonnière en Ontario grâce à l'apprentissage machine. Relevé des maladies transmissibles au Canada 2020;46(6):218-24. https://doi.org/10.14745/ccdr.v46i06a07f

Mots-clés : surveillance des maladies, apprentissage automatique, apprentissage statistique, cryptosporidiose, réseau neuronal artificiel, SARIMA, prévisions, séries chronologiques saisonnières 


\section{Introduction}

La cryptosporidiose est une maladie diarrhéique potentiellement mortelle qui touche les humains et les animaux. Elle est causée par le parasite protozoaire Cryptosporidium sp. (1). Une vingtaine des 26 espèces connues ont été associées à des infections humaines (2). La majorité des infections humaines sont causées par le $C$. hominis et le $C$. parvum, qui sont principalement liées respectivement à des transmissions anthropiques et zoonotiques (3). La principale voie d'infection pour les humains est la consommation (notamment pendant la baignade) d'eau contaminée par les oocystes des parasites.

La cryptosporidiose est souvent asymptomatique, mais elle peut entraîner des maladies gastro-intestinales légères à graves, voire le décès. La prévalence des infections chez les humains en Amérique du Nord varie entre $1 \%$ et $4 \%$ par année, mais peut atteindre $20 \%$ ailleurs (4). Bien que la cryptosporidiose soit probablement sous-déclarée, on sait qu'elle est plus fréquente chez les enfants et les personnes immunodéprimées. Aucun traitement prophylactique n'est disponible, ce qui fait de la préparation de la santé publique fondée sur la surveillance une option préventive importante.

De nouvelles possibilités d'application des statistiques, de l'épidémiologie et de la surveillance des maladies en santé publique sont apparues au cours des deux dernières décennies depuis l'avènement des mégadonnées $(5,6)$. Gunther Eysenbach a introduit le terme " infodémiologie " pour l'utilisation des mégadonnées (plus précisément des données sur l'utilisation et le comportement dans les médias sociaux) dans la surveillance de la santé (7). Un exemple frappant d'infodémiologie est le projet Google Flu Trends, qui a prédit des éclosions régionales de grippe de 7 à 10 jours avant les méthodes de surveillance conventionnelles du Center for Disease Control and Prevention ( $C D C)$, mais qui a largement surestimé la prévalence de la grippe (8). Ce projet est un exemple manifeste des possibilités ainsi que des risques liés aux mégadonnées, qu'on appelle « l'orgueil des mégadonnées » $(8,9)$.

Les mégadonnées sont souvent caractérisées par les cinq $V$ : volume, variété, vitesse, véracité et valeur (9). L'orgueil de mégadonnées fait référence à la véracité des données. La promesse des mégadonnées est que de grandes quantités de données (volume) de différents types et de sources différentes (variété) fournissent une représentation plus complète et précise de la réalité, ce qui mène à la " santé publique de précision » (10). Toutefois, lorsque les données ne sont pas représentatives de la population d'intérêt, les inférences prédictives sont biaisées.

La surveillance des maladies donne lieu à une situation de mégadonnées en raison de la vitesse et du volume des données : les données sont constamment mises à jour et leur taille augmente. La nature dynamique des données de surveillance des maladies exige une approche automatisée de l'analyse et de la prévision. La méthode traditionnelle de modélisation statistique en série chronologique est le modèle de moyenne mobile intégrée saisonnière autorégressive (SARIMA) proposé par Box et Jenkins (11). Un algorithme d'apprentissage automatique largement utilisé pour la prévision des séries chronologiques est le réseau neuronal artificiel (RNA) (à propagation avant) (12). Nous avons appliqué ces deux approches de prévision pour prévoir l'incidence mensuelle de la cryptosporidiose en Ontario en 2017 en utilisant les données de 2005 à 2016 comme ensemble initial d'apprentissage. Nous avons comparé ces approches de prévision en utilisant l'incidence de 2017 comme données de test, avec la racine de l'erreur quadratique moyenne (REQM) et l'erreur absolue moyenne (EAM) de prévision comme mesures de précision.

Des comparaisons semblables ont été rapportées dans la documentation scientifique. Zhang et Qi (13) ont comparé le SARIMA et le RNA à l'aide de simulations et ont montré que le RNA offre toujours de meilleures prévisions que le SARIMA, lorsque les données sont prétraitées de façon appropriée. Kane et coll. (14) ont comparé les prévisions de foyers de grippe aviaire $\mathrm{H} 5 \mathrm{~N} 1$ avec le SARIMA à celles de l'algorithme de forêts d'arbres décisionnels et ont conclu que l'apprentissage automatique offre une meilleure capacité de prédiction par rapport à la modélisation des séries chronologiques. De même, dans une étude sur l'incidence de la fièvre typhoïde en Chine, Zhang et coll. ont comparé la modélisation SARIMA à trois architectures différentes de RNA; les chercheurs ont conclu que les trois algorithmes de réseau neuronal surclassent le modèle statistique (15).

Cette étude vise à comparer les deux approches utilisées pour automatiser la prévision des taux d'incidence mensuels de la cryptosporidiose en Ontario pour l'année 2017. Les objectifs spécifiques étaient (1) de comparer la précision des prévisions en utilisant la REQM; (2) de comparer les prévisions en utilisant I'EAM; et (3) de comparer visuellement les taux d'incidence prévus aux séries chronologiques observées.

\section{Méthodes}

Les données que nous avons utilisées étaient des dénombrements mensuels d'incidence de la cryptosporidiose en Ontario pour les années 2005 à 2017 tels que déclarés à Santé publique Ontario et disponibles à partir des pages d'accueil respectives (16). À des fins d'analyse, nous divisons l'ensemble de données en données d'apprentissage (incidences mensuelles de 2005 à 2016) et en données de test (incidences mensuelles de 2017).

À des fins d'exploration, nous avons signalé des fourchettes $d^{\prime}$ 'incidence moyenne annuelle et mensuelle dans les données d'apprentissage et nous avons inspecté les données avec la décomposition saisonnière et tendancielle en utilisant la méthode de Loess (STL) (17). La composante saisonnière a été 
présumée invariante dans le temps ou périodique, tandis que la composante de tendance a été trouvée en utilisant une fenêtre mobile de 73 mois, ou six ans plus un mois.

Le SARIMA (11) est un modèle de génération de données qui comprend des composantes saisonnières et des tendances. Il est utilisé pour décrire les autocorrélations dans une série chronologique et pour prédire les valeurs futures. II est décrit par l'ordre des filtres appliqués pour supprimer les composantes saisonnières et de tendance ainsi que par l'ordre des corrélations décalées dans la série filtrée. La série filtrée est supposée être une fonction stationnaire et gaussienne. On résume le SARIMA comme suit : SARIMA $(p, d, q)(P, D, Q)$ s' où $S$ désigne la durée de la saison (ici 12 mois), $d$ et $D$ désignent les filtres de différence non saisonnière et saisonnière pour éliminer les composantes tendancielles et saisonnières, respectivement. De plus, $p$ et $P$ sont des ordres des paramètres d'autocorrélation non saisonniers et saisonniers, respectivement. Enfin, $q$ et $Q$ désignent l'ordre non saisonnier et saisonnier des paramètres de moyenne mobile. L'approche de modélisation SARIMA a été automatisée en utilisant l'estimation du maximum de vraisemblance et la sélection progressive rétrospective du modèle avec le critère d'information bayésienne (BIC). Le SARIMA, adapté aux données d'apprentissage 2005 à 2016, a ensuite été utilisé pour prévoir les incidences mensuelles pour les données de test de 2017.

Le RNA est un algorithme automatisé et axé sur les données qui permet de prévoir les données des séries chronologiques. Bien qu'une variété d'architectures de RNA existe $(18,19)$, nous avons appliqué le réseau neuronal multicouche de base, à propagation avant, avec une seule couche cachée dans cette étude (12). Plus précisément, le RNA est décrit comme RNA $(p, P, k)$, où $p, P$ et $S$ ont les mêmes significations que dans le modèle SARIMA, et $k$ indique le nombre de nœuds dans la couche cachée. La sélection automatique des valeurs d'ordre du RNA était la suivante : $S=12$ est connu; $k$ était la valeur arrondie de $(p+P+1) / 2$, où $P$ était réglé à $P=1$ pour tenir compte de la saisonnalité linéaire; et $p$ a été sélectionné comme l'ordre optimal d'un modèle autorégressif convenant au reste du terme de la série décomposée de la STL.

Nous avons appliqué l'algorithme du RNA comme suit : les combinaisons linéaires de données d'entrée ont été soumises à la fonction d'activation sigmoïde non linéaire $1 /(1+\exp [-z])$ comme sortie d'une couche cachée, et les résultats de la couche cachée ont ensuite été agrégés sous forme de combinaisons linéaires, ce qui a donné lieu à la sortie finale. Le RNA a été formé en utilisant 100 répétitions, c'est-à-dire 100 valeurs de départ aléatoires différentes pour les paramètres de poids des combinaisons linéaires entre les couches d'entrée et cachées, ainsi que les couches cachées et de sortie. De plus, la série d'entrées (c.-à-d. les données de 2005 à 2016) a été prétraitée au moyen d'une sélection automatique du paramètre de transformation de Box-Cox (par la méthode Guerrero (12)) suivie d'une studentisation (c.-à-d. le centrage et la mise à l'échelle). Pour chaque répétition, l'algorithme a été entraîné par un processus expérimental itératif d'optimisation d'une fonction de perte. L'ensemble des prévisions qui en a résulté a été moyennée sur toutes les itérations.

Les deux approches de prévision fournissent des intervalles de prévision. L'intervalle de prévision du SARIMA était fondé sur l'estimation du paramètre du modèle. L'intervalle de prévision du RNA était fondé sur 1000 exemplaires de chemins générés par bootstrap (12), c'est-à-dire en utilisant des résidus passés rééchantillonnés. De plus, les deux approches de prévision ont été comparées par leurs mesures d'exactitude (REQM et EAM) pour les prévisions mensuelles et les données d'essai observées de l'année 2017.

Toutes les analyses de données ont été effectuées dans $R$ (20) et RStudio (21) à l'aide de la librairie « forecast » (12).

\section{Résultats}

La série chronologique des incidences de cryptosporidiose signalées mensuellement en Ontario pour les années 2005 à 2016 est dominée par une composante saisonnière, avec des pics en été et seulement une faible - voire aucune - tendance à la hausse (figure 1). La décomposition du STL à la figure 2 confirme cette impression. La série chronologique des données sur la formation est relativement courte, 12 ans comprenant 4152 cas, soit une moyenne annuelle de 346 cas, ce qui équivaut à environ 2,57 cas annuels pour 100000 personnes à risque. Le nombre moyen de cas mensuels était de 29, allant de 6 à 109 cas de 2005 à 2016.

\section{Figure 1 : Graphique chronologique de l'incidence mensuelle de la cryptosporidiose en Ontario de 2005 à 2016}

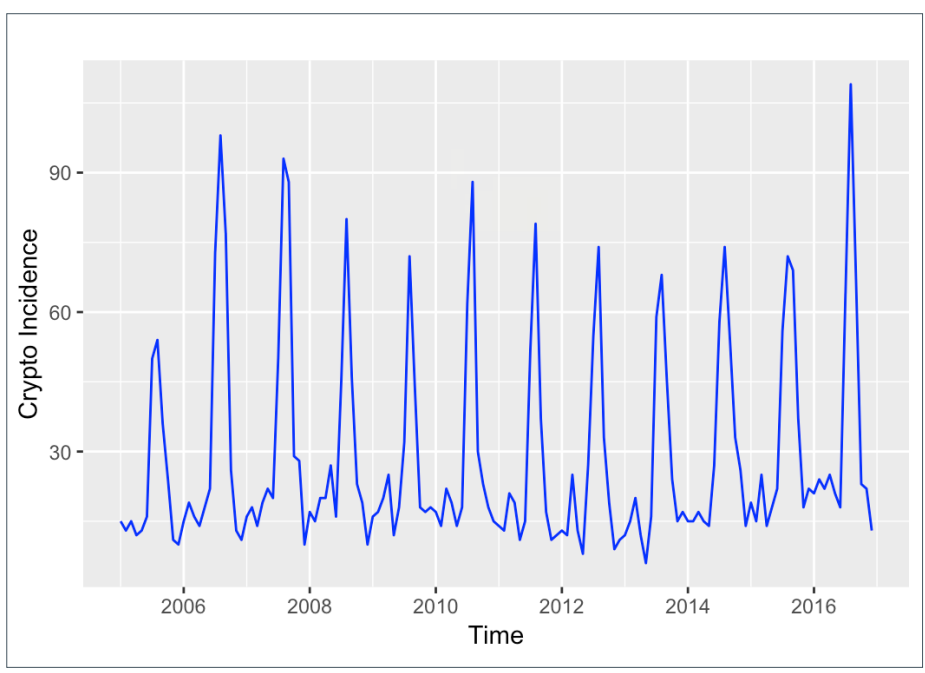

Abréviation : Crypto, cryptosporidiose (Figure en anglais seulement) 
Figure 2 : Série chronologique des incidences mensuelles de la cryptosporidiose de 2005 à $2016^{a}$

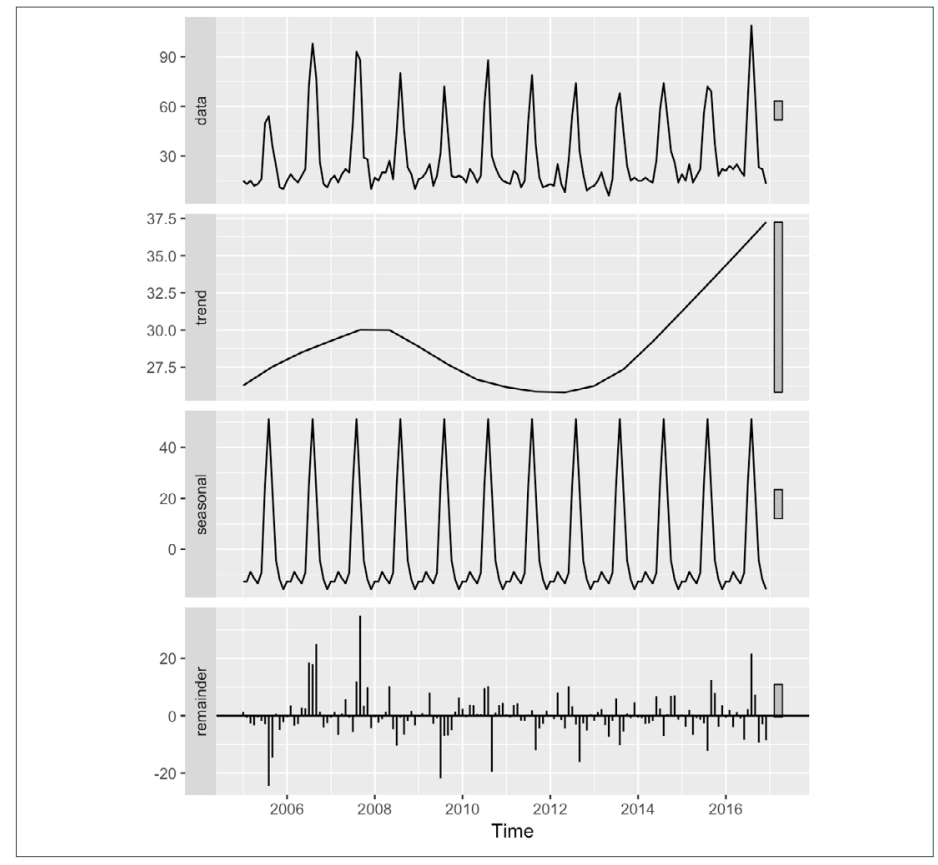

a Décomposition saisonnière et tendance basée sur le tracé de la procédure de STL (procédure Loess) de l'ensemble de données de formation (17)

(Figure en anglais seulement)

La sélection automatisée par étapes du modèle a abouti à un SARIMA $(1,0,0)(1,1,0)_{12}$ dont les estimations des paramètres du modèle sont le paramètre autorégressif du premier ordre $A R$ $(1)=0,41$ (erreur standard $[S E]=0,08$ ) et le paramètre autorégressif saisonnier du premier ordre $\operatorname{SAR}(1)=0,35(S E=0,10)$ (figure 3 ).

Le RNA sélectionné automatiquement est d'ordre RNA $(11,1,6)_{12^{\prime}}$ c.-à-d. que les 11 dernières observations plus la première observation saisonnière sont linéairement combinées en six nœuds d'une seule couche cachée. La série d'entrée a été transformée selon la méthode de Box-Cox avec un paramètre choisi automatiquement $\lambda=0,21$. Les prévisions du RNA sont visualisées avec des intervalles de prévision de $80 \%$ et $95 \%$ à la figure 4 .

Les incidences mensuelles observées et les prévisions arrondies sont présentées au tableau 1 et à la figure 5 pour les deux modèles. Le tableau 2 présente les résumés du REQM et de I'EAM tirés des prévisions de 2017 pour les deux approches.
Figure 3 : Prévisions des incidences mensuelles de cryptosporidiose de 2017 avec des intervalles de prévision de $80 \%$ et $95 \%$ à partir d'un SARIMA $(1,0,0)$ $(1,1,0)_{12}$

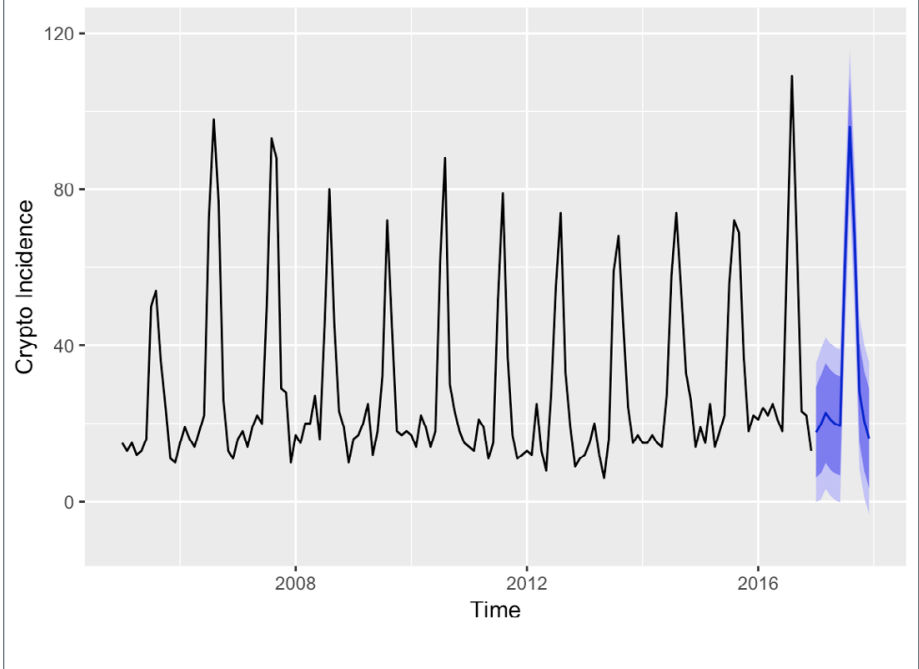

Abréviations : Crypto, cryptosporidiose; SARIMA, moyenne mobile intégrée saisonnière autorégressive

(Figure en anglais seulement)

Figure 4 : Prévisions des incidences mensuelles de cryptosporidiose de 2017 avec des intervalles de prévision par bootstrap à $80 \%$ et $95 \%$ à partir d'un RNA $(11,1,6)_{12}$

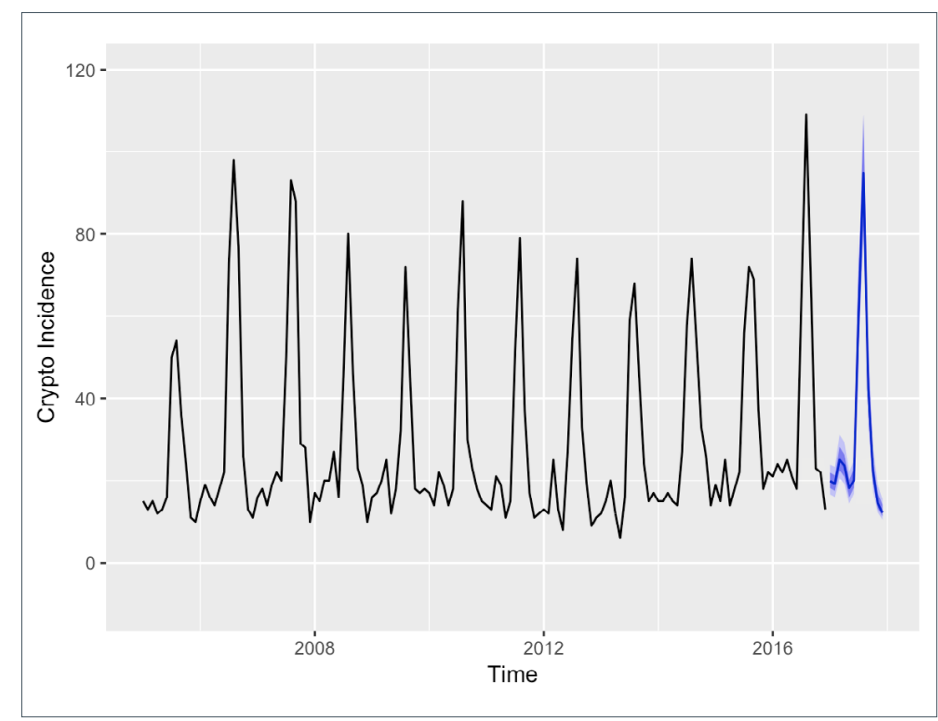

Abréviations : Crypto, cryptosporidiose; RNA, réseau neuronal artificiel (Figure en anglais seulement)

Tableau 1 : Taux d'incidence observés de la cryptosporidiose pour 2017 et prévisions arrondies de l'approche SARIMA et du RNA ${ }^{a}$

\begin{tabular}{|c|c|c|c|c|c|c|c|c|c|c|c|c|}
\hline \multicolumn{13}{|c|}{ Taux d'incidence par mois } \\
\hline Mois & janv. & févr. & mars & avril & mai & juin & juill. & août & sept. & oct. & nov. & déc. \\
\hline Observé & 23 & 17 & 25 & 22 & 16 & 24 & 48 & 70 & 55 & 32 & 33 & 20 \\
\hline SARIMA & 18 & 20 & 23 & 21 & 20 & 19 & 61 & 96 & 68 & 28 & 21 & 16 \\
\hline RNA & 20 & 19 & 25 & 24 & 18 & 20 & 62 & 95 & 42 & 22 & 15 & 12 \\
\hline
\end{tabular}

Abréviations : RNA, réseau neuronal artificiel; SARIMA, moyenne mobile intégrée saisonnière autorégressive

a Pour chaque mois, les prévisions les plus proches de l'incidence observée sont en caractères gras 
Figure 5: Graphique chronologique des incidences mensuelles de cryptosporidioses observées pour 2017 et des prévisions de l'approche SARIMA et du RNA

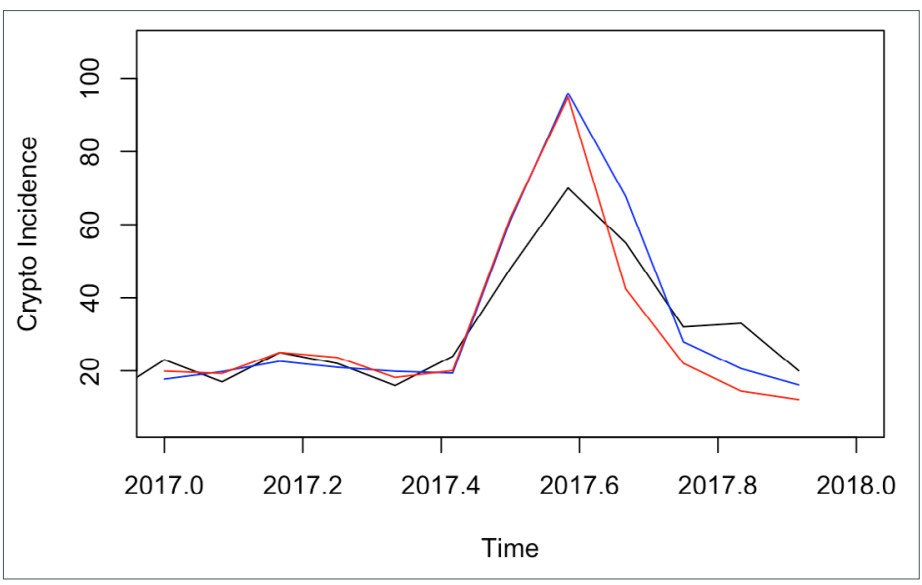

Abréviations : Crypto, cryptosporidiose (ligne noire); RNA, réseau neuronal artificiel (ligne rouge); SARIMA, moyenne mobile intégrée saisonnière autorégressive (ligne bleue)

(Figure en anglais seulement)

Tableau 2 : Mesures de performance prédictive pour I'approche SARIMA et le RNA

\begin{tabular}{|l|r|r|}
\multicolumn{1}{|c|}{ Modèle } & REQM & EAM \\
\hline SARIMA $(1,0,0)(1,1,0)_{12}$ & 10,3 & 7,7 \\
\hline RNA $(11,1,6)_{12}$ & 11,2 & 8,4 \\
\hline
\end{tabular}

Abréviations : EAM, erreur absolue moyenne; REQM, racine de l'erreur quadratique moyenne; RNA, réseau neuronal artificiel; SARIMA, moyenne mobile intégrée saisonnière autorégressive

\section{Discussion}

L'incidence mensuelle de la cryptosporidiose en Ontario est caractérisée par une tendance saisonnière dominante qui culmine généralement en août. Le court pic d'incidence peut appuyer le concept du comportement humain comme principal facteur d'infection puisque les conditions environnementales (e.g. la température ambiante) ne varient pas de la même façon que l'incidence. Aucune tendance à la hausse n'a été relevée, ce qui signifie que l'incidence n'est pas émergente.

Ni l'algorithme d'apprentissage automatique (c.-à-d. le RNA) ni la méthode d'apprentissage statistique (c.-à-d. le SARIMA) n'ont obtenu un rendement supérieur pour ce qui est de prédire l'incidence mensuelle de la cryptosporidiose. Alors que les prévisions du RNA étaient plus proches des observations pour six mois, le SARIMA a obtenu de meilleurs résultats pour un groupe différent de cinq mois; les deux méthodes étaient à égalité pour le mois de septembre 2017 (voir le tableau 1). Toutefois, les mesures de l'exactitude REQM et EAM indiquent un léger avantage pour les prévisions du SARIMA : la REQM du RNA et I'EAM étaient supérieurs de 0,9 et 0,7 unité, respectivement (voir le tableau 2).

Ce léger avantage pour le SARIMA est interprété comme suit : les prévisions du SARIMA sont, en moyenne, presque un cas par mois plus précises que les prévisions du RNA. Bien que ce résultat soit inattendu en ce qui concerne les rapports cités (13-15), il est conforme à un examen systématique (22) qui n'a trouvé aucune preuve de prédictions plus exactes des solutions de rechange à l'apprentissage machine à la modélisation de régression logistique statistique. Toutefois, il convient de noter qu'il s'agit d'une étude de cas et que les résultats sont propres à cet exemple. Bien que le SARIMA suppose des résidus de bruit blanc et une composante saisonnière additive, cela n'a pas été vérifié ici à l'aide de l'approche de modélisation automatisée. De même, le RNA est optimisé à l'aide de la rétropropagation, qui est connue pour avoir de la difficulté à trouver les estimations de paramètres optimales (19). Par conséquent, le RNA utilise les prévisions d'ensemble pour se prémunir contre les prévisions erronées individuelles.

Un prétraitement adéquat des données est important pour les algorithmes d'apprentissage automatique (23). Cela signifie qu'une série chronologique doit être mise à l'échelle et centrée (c.-à-d. studentisée ou normalisée) avant l'analyse. Le prétraitement des données est une partie naturelle de l'approche de modélisation de la moyenne mobile intégrée autorégressive, car les effets saisonniers et les effets de tendance sont filtrés avant que le modèle ne soit adapté à la série chronologique. Dans notre étude, la sélection progressive du modèle a permis de filtrer un effet saisonnier, mais aucun effet de tendance n'a été identifié ou supprimé. Le RNA a été prétraité par une transformation Box-Cox, suivie d'un centrage et d'une mise à l'échelle.

L'analyse des mégadonnées est souvent présentée avec des algorithmes d'apprentissage automatique pour l'inférence, c.-à-d. la modélisation prédictive. Cela pourrait s'expliquer par l'impression que les méthodes statistiques traditionnelles ne conviennent pas aux défis des mégadonnées. Par exemple, la variété des données exprimées par le nombre de covariables pourrait rendre l'inférence statistique traditionnelle moins attrayante et peu pratique. Par ailleurs, les algorithmes d'apprentissage automatique sont conçus autour de méthodes statistiques modernes pour la réduction des dimensions et la régularisation (e.g. régression Lasso). La phase d'entraînement des algorithmes d'apprentissage automatique est ce qu'on appelle aussi l'estimation des paramètres dans la modélisation statistique et elle n'est pas différente des méthodes d'apprentissage statistique, car elle est fondée sur la validation croisée et le bootstrap.

En résumé, dans une certaine mesure, l'apprentissage statistique et l'apprentissage automatique ne diffèrent pas. Toutefois, dans le domaine de la santé publique, les applications de l'analyse des mégadonnées, notamment la modélisation prédictive, y compris la prévision des séries chronologiques, diffèrent de l'analyse traditionnelle des données biostatistiques en ce qui concerne l'identification et l'évaluation des facteurs de risque. Breiman a fait la distinction entre "les deux cultures » de la modélisation statistique, soit la culture de la modélisation des 
données et la culture de la modélisation algorithmique (24). II a soutenu que la théorie statistique n'est pas pertinente si les hypothèses de modélisation ne sont pas respectées dans des situations de données réelles. Cependant, il a également admis que les algorithmes d'apprentissage automatique sont souvent basés sur peu de théorie, et que les hypothèses de modélisation sont remplacées par les propriétés des algorithmes, c.-à-d. si ces derniers convergent et produisent de bonnes prévisions.

D'un point de vue philosophique, l'apprentissage automatique est basé sur une "boîte noire " qui n'est pas ouverte aux interprétations ou aux explications. Dans l'exemple actuel, I'algorithme du RNA $(11,1,6)_{12}$ comprenait une combinaison non linéaire des données de la série chronologique et 85 paramètres (23). Par ailleurs, le SARIMA décrit comment les observations antérieures influent sur le déroulement futur d'un processus; cette caractéristique pourrait suggérer des hypothèses causales (25). Par conséquent, il n'est pas tout à fait correct de simplement comparer les méthodes de prévision à leurs valeurs prévues ou à leurs mesures de précision, car les approches sont différentes sur le plan philosophique et ne sont pas entièrement comparables; le RNA est un algorithme prédictif, tandis que le SARIMA est un modèle descriptif et prédictif.

\section{Limites}

Une des limites de cette étude est le manque d'ajustement pour la population à risque. En effet, la population de l'Ontario augmente régulièrement, mais à un taux annuel inférieur à 0,5\%, ce qui est négligeable dans ce contexte, où la sous-déclaration est plus préoccupante. Aucune tendance des taux mensuels d'incidence de la cryptosporidiose n'a été indiquée par l'approche du SARIMA ou le RNA.

\section{Conclusion}

La cryptosporidiose est une maladie fortement saisonnière qui entraîne des périodes favorables et défavorables pour la santé publique. Les méthodes d'apprentissage automatique convenant à la prévision des données chronologiques de la santé publique provenant des systèmes de surveillance sont de plus en plus populaires; il a été démontré qu'elles sont plus précises que les méthodes statistiques traditionnelles. Toutefois, dans cette étude de cas en particulier, le SARIMA a donné lieu à des REQM et à des EAM légèrement inférieures et donc à une plus grande exactitude que le RNA. Les deux approches de prévision ont bien saisi la tendance saisonnière de la cryptosporidiose.

Les études futures devraient utiliser des algorithmes supplémentaires (e.g. forêts d'arbres décisionnels) et évaluer l'exactitude dans différents contextes, soit en utilisant d'autres maladies pour les études de cas, soit en utilisant une approche plus systématique en réalisant des études de simulation.

\section{Déclaration des auteurs}

O. B. a conçu l'examen, recueilli les données et effectué l'analyse des données

O. B., L. T. W. et S. D. M. ont tous écrit et approuvé le manuscrit.

\section{Conflit d'intérêts}

Les auteurs déclarent n'avoir aucun conflit d'intérêts.

\section{Références}

1. Abeywardena $H$, Jex AR, Gasser RB. A perspective on Cryptosporidium and Giardia, with an emphasis on bovines and recent epidemiological findings. Adv Parasitol 2015:88:243-301. DOI PubMed

2. Ryan U, Fayer R, Xiao L. Cryptosporidium species in humans and animals: current understanding and research needs. Parasitology 2014:141(13):1667-85. DOI PubMed

3. Nwosu A, Berke O, Pearl DL, Trotz-Williams LA . Exploring the geographical distribution of cryptosporidiosis in the cattle population of Southern Ontario, Canada, 2011-2014. Geospat Health 2019;14(2);236-46. DOI

4. Medema G, Teunis P, Blokker M, Deere D, Davison A, Charles $\mathrm{P}$, Loret JF. Risk assessment of Cryptosporidium in drinking water. Geneva $(\mathrm{CH})$ : World Health Organization; 2009. pp. 143.

5. Mooney SJ, Pejaver V. Big data in public health: terminology, machine learning and privacy. Annu Rev Public Health 2018;39:95-112. DOI PubMed

6. $\mathrm{Bi} \mathrm{Q}$, Goodman KE, Kaminsky J, Lessler J. What is machine learning? A primer for the epidemiologists. Am J Epidemiol 2019;188(12):2222-39. DOI PubMed

7. Eysenbach G. Infodemiology: the epidemiology of (mis) information. Am J Med 2002;113(9):763-5. DOl PubMed

8. Lazer D, Kennedy R, King G, Vespignani A. Big data. The parable of Google Flu: traps in big data analysis. Science 2014 Mar;343(6176):1203-5. DOI PubMed

9. Fuller D, Buote R, Stanley K. A glossary for big data in population and public health: discussion and commentary on terminology and research methods. J Epidemiol Community Health 2017 Nov;71:1113-7. DOI PubMed

10. Dowell SF, Blazes D, Desmond-Hellmann S. Four steps to precision public health. Nature 2016;540:189-91. DOI

11. Box G, Jenkins G. Time series analysis: forecasting and control. San Francisco: Holden-Day; 1970.

12. Hyndman RJ, Athanasopoulos G. Forecasting: principles and practice (2nd ed). Melbourne (AU): OTexts; 2018.

13. Zhang GP, Qi M. Neural network forecasting for seasonal and trend time series. Eur J Oper Res 2005;160(2):501-14. DOI 
14. Kane MJ, Price N, Scotch M, Rabinowitz P. Comparison of ARIMA and Random Forest time series models for prediction of avian influenza H5N1 outbreaks. BMC Bioinformatics 2014;15:276. DOI PubMed

15. Zhang $X$, Liu Y, Yang $M$, Zhang T, Young AA, Li X. Comparative study of four time series methods in forecasting typhoid fever incidence in China. PLoS One 2013 May;8(5):e63116. DOI PubMed

16. Santé publique Ontario. Tendances des maladies infectieuses en Ontario. Toronto (ON) : Santé publique Ontario ; 2020 (accédé 2020-01-20). https:// www.publichealthontario.ca/fr/data-and-analysis/ infectious-disease/reportable-disease-trends-annually\#/14

17. Cleveland RB, Cleveland WS, McRae JE, Terpenning I. STL: a seasonal-trend decomposition procedure based on Loess. J Off Stat 1990;6(1):3-73.

18. Jain AK, Mao J, Mohluddin KM. Artificial neural networks: a tutorial. Computer 1996;29(3):31-4. DOI
19. Warner B, Misra M. Understanding neural networks as statistical tools. Am Stat 1996;50(4):284-92. DOI

20. R Core Team. R: A language and environment for statistical computing. Vienna (AT): R Foundation for Statistical Computing; 2019.

21. RStudio Team. RStudio: integrated development for R. Boston (MA): RStudio, Inc.; 2018.

22. Christodoulou E, Ma J, Collins GS, Steyerberg EW, Verbakel JY, Van Calster B. A systematic review shows no performance benefit of machine learning over logistic regression for clinical prediction models. J Clin Epidemiol 2019 Jun;110:12-22. DOl PubMed

23. Kuhn M, Johnson K. Applied predictive modeling. New York (NY): Springer; 2013. pp. 1-600. DOI

24. Breiman L. Statistical modeling: the two cultures. Stat Sci 2001;16(3):199-231. DOI

25. Shmueli G. To explain or to predict? Stat Sci 2010;5(3): 299-310. DOI 